\title{
Thermal coupling between a spiral pipe and a conducting volume
}

\author{
M. Alalaimi ${ }^{\mathrm{a}}$, S. Lorente $^{\mathrm{b}}$, A. Bejan ${ }^{\mathrm{a}, *}$ \\ ${ }^{a}$ Duke University, Department of Mechanical Engineering and Materials Science, Durham, NC 27708-0300, USA \\ ${ }^{\mathrm{b}}$ Université de Toulouse, UPS, INSA, LMDC (Laboratoire Matériaux et Durabilité des Constructions), 135, avenue de Rangueil, F-31 077 Toulouse Cedex 04, France
}

\section{A R T I C L E I N F O}

\section{Article history:}

Received 26 February 2014

Accepted 28 April 2014

Available online 5 June 2014

\section{Keywords:}

Constructal design

Cooling

Heat pumps

Spiral

Ground heat exchangers

\begin{abstract}
A B S T R A C T
Here we document the effect of flow configuration on the heat transfer performance of a spiral shaped pipe embedded in a cylindrical conducting volume. We considered several configurations with fixed volumes of fluid and solid. First, we optimized the geometry of two spiral pipes with varying spacing between the spiral turns and the vertical spacing between the two spirals. Next, we extended the method to three spiral pipes with varying the spacing between the spiral turns and the spacing between the spiral pipes, and changing the dimensions of the conducting volume. We found that optimal spacings between the spiral turns and spire planes exist, such that the volumetric heat transfer rate is maximal. The optimized features of the heat transfer architecture are robust.
\end{abstract}

(c) 2014 Elsevier Ltd. All rights reserved.

\section{Introduction}

In nature, anything that moves relative to a reference point might be considered as a flow system, whether this flow is animate such as the human lung and the blood vascular system in the human body, or inanimate such as the river basins and deltas. The generation of flow configuration in nature and engineering is governed by the constructal law. Any flow system that occurs in nature generates structure and shape over time in order to facilitate movement and overcome flow resistance. Thermodynamically, resistances are imperfections (sources of irreversibility) distributed over the flow volume. Imperfections which cannot be eliminated, but can be redistributed in order to reduce the global resistance. The only way to redistribute the imperfections is to vary the flow geometry. Therefore, the geometry of all the flow designs that occur in nature and engineering is the governing factor in design performance [1-3].

Heating and air conditioning are technologies that have played a key role in increasing economic activity during the past century [1]. Regions of the globe historically sleepy because of extreme warmth or cold were brought to "temperate" climate, life style and productivity by this technology. Today, this technology is so prevalent and so useful that it is taken for granted. Geothermal heat pumps have grown rapidly in many countries since 1980s. One design consists of using a borehole heat pump, which can be utilized economically by drawing heat from low temperature

\footnotetext{
* Corresponding author. Tel.: +1 919660 5309; fax: +1 9196608963.

E-mail address: dalford@duke.edu (A. Bejan).
}

sources [4-22]. There are two types of ground heat exchangers: open and closed. The open system is a direct heat exchanger between the ground and the medium. The closed system is of the indirect type, with pipes buried horizontally or vertically. The choice of horizontal versus vertical ground heat exchangers depends on the condition of the surrounding soil.

In this paper we consider the fundamental configuration of time-dependent heat transfer between spiral pipes and a conducting volume that functions as heat sources or sinks. The objective is to find the optimum spacing between spiral pipes, and the spacing between spiral turns. The fundamental aspect is the focus on the relation between the morphing of the system configuration and the improvements in the global performance of the complex flow system, in accord with constructal design.

\section{Model}

Consider the heat transfer performance of an embedded heat exchanger shaped as an Archimedean spiral. The heat flow system has two domains, one fluid and the other solid: an isothermal spiral pipe with fixed diameter $D_{0}$ and length $L_{s}$, and a fixed solid volume with cylindrical shape of diameter $D$ with height $H=D / 2$, as shown in Figs. 1 and 2. The spiral polar coordinates $r$ is defined as

$r=a+S_{x} \theta$

where $r=a$ is the starting point of the spiral, $S_{x}$ is the spacing between successive turns, and $\theta$ is the angular position expressed in radians. 


\section{Nomenclature}

$A_{c} \quad$ cylinder cross-sectional area, $\mathrm{m}^{2}$

$A_{s} \quad$ spiral cross-sectional area, $\mathrm{m}^{2}$

$S_{x} \quad$ spacing between turns, $m$

$D \quad$ diameter of area, $m$

$D_{0} \quad$ pipe diameter, $\mathrm{m}$

$H$ height, $\mathrm{m}$

$L_{s} \quad$ spiral length, $\mathrm{m}$

$n$ number of turns

$r \quad$ radial coordinate, $m$

$S_{z} \quad$ spacing between the two spirals, $\mathrm{m}$

$t \quad$ time, $s$

$T_{c} \quad$ initial temperature, $\mathrm{K}$

$T_{s} \quad$ pipe temperature, $\mathrm{K}$
$V_{c} \quad$ volume of cylinder, $\mathrm{m}^{3}$

$V_{s} \quad$ volume of spiral, $\mathrm{m}^{3}$

$z \quad$ coordinate, $\mathrm{m}$

Greek symbols

$\alpha \quad$ thermal diffusivity, $\mathrm{m}^{2} / \mathrm{s}$

$\theta \quad$ angle, rad

$\varepsilon \quad$ dimensionless spacing $S_{x} / D$

$\zeta$ dimensionless $S_{z} / H$

Subscripts

* dimensionless

avg volume averaged
In order to determine how much material is needed to make the spiral, imagine that the spacing between the successive turns $\left(S_{x}\right)$ is very small. The spiral would occupy a disk with radius equal to the number of turns $(n)$ multiplied by the spacing $\left(S_{x}\right)$, so the spire cross-sectional area is $A_{s}=\pi n^{2} S_{x}^{2}$. The cross-sectional area also equals to the spiral length $\left(L_{s}\right.$ multiplied by the spacing between the turns $\left(S_{x}\right)$, namely $A_{S}=L_{S} S_{X}$, therefore the spiral length is approximately $L_{s} \approx \pi S_{x} n^{2}$. Two spiral pipes are buried in a solid body with cylindrical shape of volume $V_{c}=A_{c} \times H=\frac{\pi}{4} D^{2} H$, where $A_{c}$ is the cylinder cross-sectional area. The boundary of the solid cylindrical volume is insulated. The initial temperature of the body $\left(T_{c}\right)$ is higher than the temperature of the spiral pipe $\left(T_{s}\right)$.

Transient heat conduction in the cylindrical volume was simulated using a finite element software package [23]. The conservation of energy in the solid is governed by

$\frac{\partial T}{\partial t}=\alpha\left[\frac{1}{r} \frac{\partial}{\partial r}\left(r \frac{\partial T}{\partial r}\right)+\frac{1}{r^{2}} \frac{\partial^{2} T}{\partial \theta^{2}}+\frac{\partial^{2} T}{\partial z^{2}}\right]$

where $r, \theta$ and $z$ are defined in Fig. 2, and $\alpha$ is the thermal diffusivity of the solid.

Eq. (2) can be written in dimensionless form by using the dimensionless variables
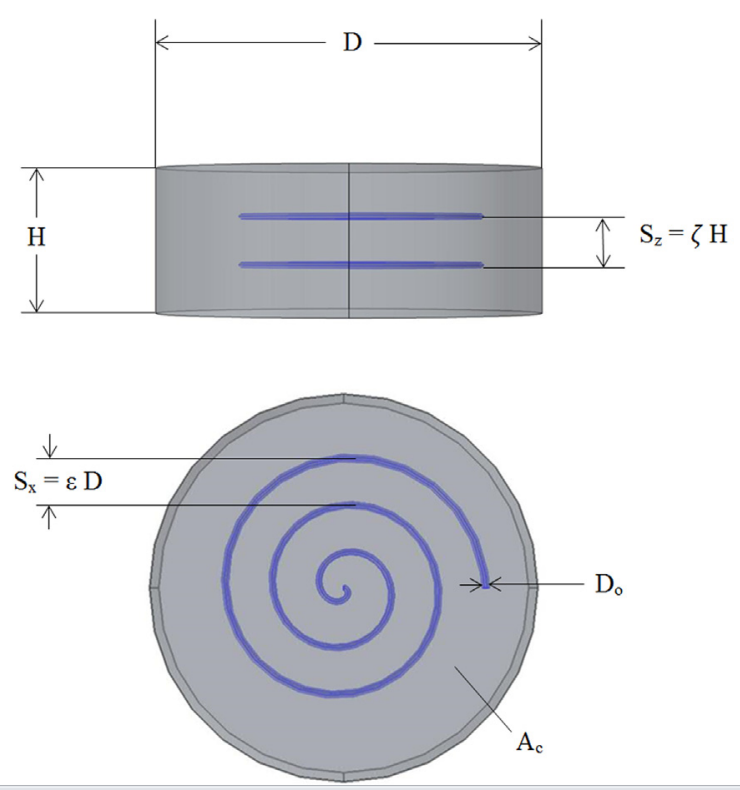

Fig. 1. The geometry of spiral pipes embedded in a cylindrical volume.
$r_{*}=\frac{r}{D}, \quad z_{*}=\frac{z}{D}$

$t_{*}=\frac{\alpha t}{D^{2}}, \quad T_{*}=\frac{T-T_{s}}{T_{c}-T_{s}}$

$\frac{\partial T_{*}}{\partial t_{*}}=\frac{1}{r_{*}} \frac{\partial}{\partial \mathbf{r}_{*}}\left(r_{*} \frac{\partial T_{*}}{\partial r_{*}}\right)+\frac{1}{r_{*}^{2}} \frac{\partial^{2} T_{*}}{\partial \theta_{*}^{2}}+\frac{\partial^{2} T_{*}}{\partial z_{*}^{2}}$

Eq. (4) was subjected to the initial and boundary conditions

$T_{*}=1$ at $t_{*}=0$

$\frac{\partial T_{*}}{\partial r_{*}}=0$ at $r_{*}=-1,1$

$\frac{\partial T_{*}}{\partial z_{*}}=0$ at $z_{*}=-1,1$

\section{Numerical method}

Heat flows from the cylindrical volume to the embedded pipes. The average temperature of the cylindrical volume decreases with time, and it approaches the pipe temperature $T_{S}\left(\right.$ or $T_{*}=0$ ), which is fixed. The spacing between the spiral turns $S_{x}$ can be related to the diameter of the cylinder $(D)$ as follows

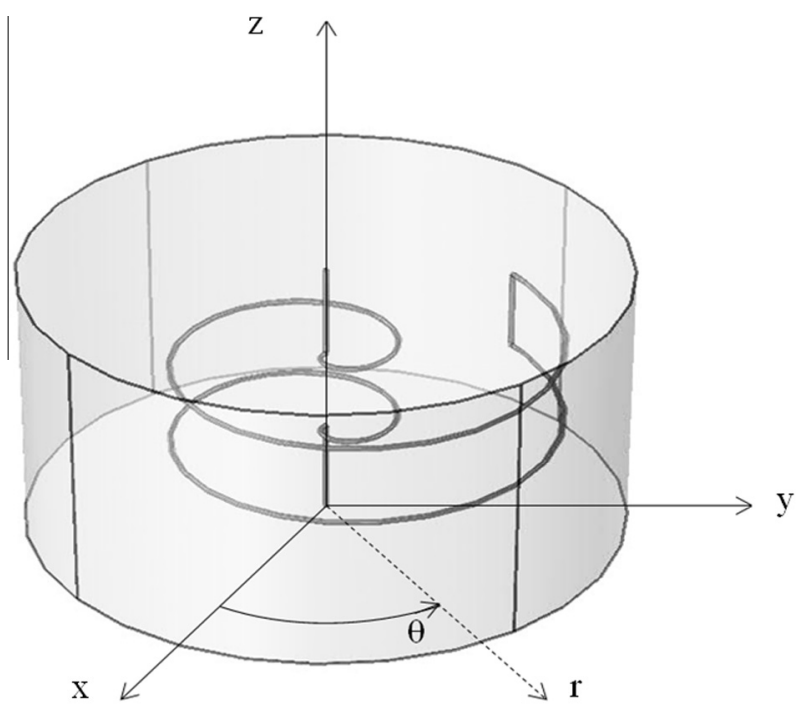

Fig. 2. The coordinates of the flow system of Fig. 1. 
$S_{x}=\varepsilon D, \quad D_{0}<S_{x}<\frac{1}{2}\left[\frac{D}{n}-n D_{0}\right]$

where $\varepsilon$ is a dimensionless factor smaller than 1 , which varies as the spacing between the turns varies. The spacing between the spiral turns can be written in dimensionless form as

$S_{X_{*}}=\frac{S_{x}}{D}=\varepsilon$

Similarly, the spacing between the planes of the two spirals $S_{z}$ can be related to the height of the cylinder $(H)$ as follows

$S_{z}=\zeta H, \quad D_{0}<S_{z} \frac{H}{2}-D_{0}$

where $\zeta$ is a dimensionless factor smaller than 1 . The dimensionless spacing between the two spirals is

$S_{z *}=\frac{S_{z}}{D}=\zeta \frac{H}{D}$

The diameter and height of the cylinder are fixed and can be expressed in dimensionless form, $D_{*}=D / D=1$, and $H_{*}=H / D=1 / 2$. The dimensionless spacings become

$S_{X_{*}}=\varepsilon D_{*}, \quad S_{X_{*}}=\zeta H_{*}$

The total length of the spiral is fixed and assumed to be equal to twice the diameter of the cylinder $L_{s}=2 D$, or in dimensionless form $L_{S *}=2$.

The governing Eqs. (2)-(7) were solved numerically using the software COMSOL [23]. In order to test the accuracy of the grid using a free tetrahedral mesh, lets pick values for $\varepsilon=0.2, \zeta=0.2$ and fix the duration of the heat transfer process at $t_{*}=0.3$. Table 1 shows the accuracy test for the grid, starting with a coarse mesh proceeding to an extremely fine mesh. In the present numerical study, the results for this problem are grid independent and the meshing scheme is free tetrahedral. All the simulations in this problem are done with extremely fine mesh identified in Table 1.

\section{The effect of flow geometry}

Fig. 3 shows the variation of the volume-averaged temperature $T_{*, a v g}$ with time. The spacing between the spiral turns $\varepsilon$ is fixed. We document the effect of the spacing between the two spirals $\zeta$ on the average temperature over time. As the dimensionless factor $\zeta$ increases, the curves become steeper. The effect of $\zeta$ weakens as $\zeta$ becomes greater than 0.3 .

We are interested in finding the optimum spacings ( $\varepsilon$ and $\zeta$ ) that correspond to minimum average temperature of the cylindrical volume $V_{c}$. For this purpose we fixed the duration of the heat transfer process at $t_{*}=0.3$, and investigated the effect of the spacing $\varepsilon$ on the average temperature $T_{*}$.

Fig. 4 shows the effect of varying the spacing between the spiral turns $\varepsilon$ on the average temperature of the cylindrical volume for three different values of $\zeta$. There is an optimum spacing between the spiral turns $\left(\varepsilon_{o p t}=0.3\right.$, independent of $\zeta$ ) that corresponds to the minimum average temperature of the cylindrical volume. By repeating the work of Fig. 4 for $t_{*}$ values above and below 0.3,

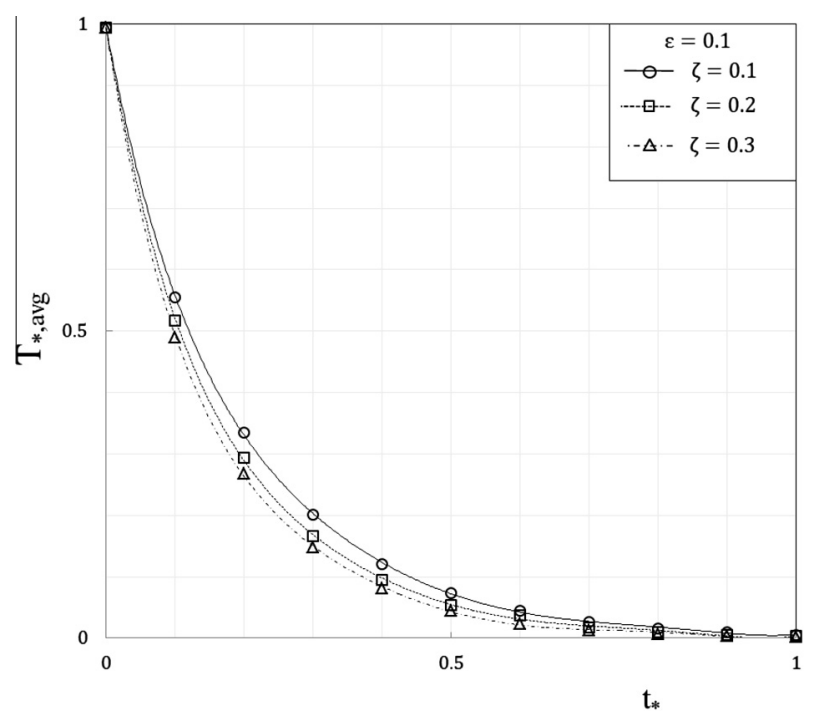

Fig. 3. The variation of the average dimensionless temperature with time.

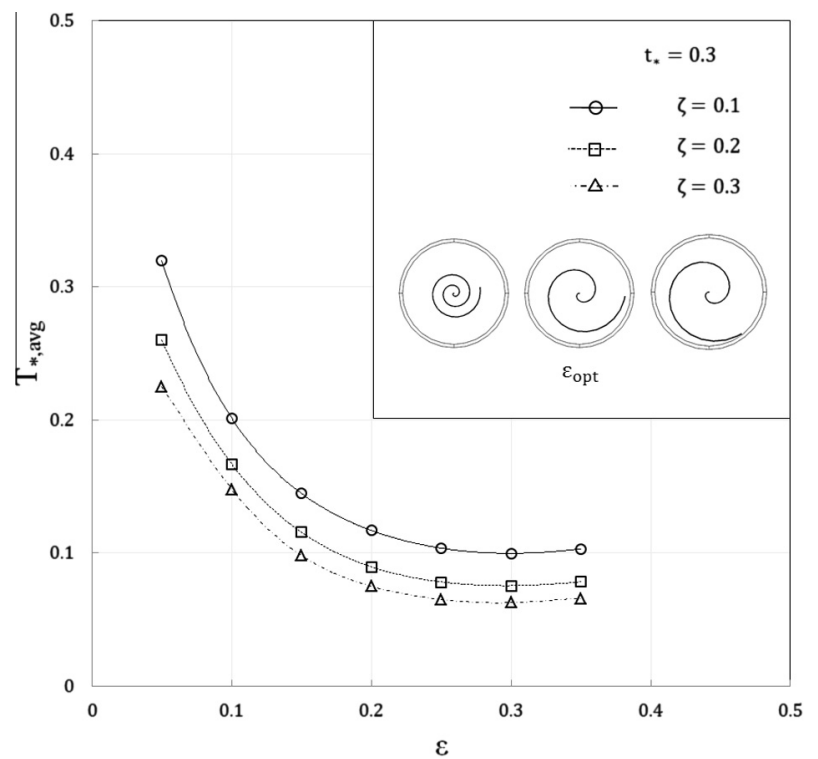

Fig. 4. The effect of the spacing between the spiral turns $(\varepsilon)$ on the average temperature of the cylindrical body.

we found that the optimum spacing $\varepsilon_{\mathrm{opt}}$ is independent of the time $t_{*}$. In all these cases, the changes in the vertical spacing $\zeta$ do not affect the value of the optimum spacing between the spiral turns $\varepsilon_{o p t}$.

The effect of the spacing between the two spirals on the average temperature can be determined by varying the value of $\zeta$ after fixing the value of $\varepsilon$ to $\varepsilon_{o p t}$, as shown in Fig. 5. The optimum value of $\zeta$ that corresponds to the minimum average temperature of the cylindrical volume is $\zeta_{\text {opt }}=0.5$. By repeating these simulations for

Table 1

Mesh accuracy test.

\begin{tabular}{|c|c|c|c|c|}
\hline & Number of elements & Mean element size & $T_{*, a v g}$ & Difference (\%) \\
\hline Coarse & 38,912 & 0.156 & 0.0840 & - \\
\hline Fine & 104,782 & 0.091 & 0.0903 & 7.48 \\
\hline Finer & 454,162 & 0.065 & 0.0895 & 0.92 \\
\hline Extra fine & $3,181,616$ & 0.044 & 0.0895 & 0.045 \\
\hline Extremely fine & $9,232,970$ & 0.026 & 0.0895 & $\approx 0.001$ \\
\hline
\end{tabular}




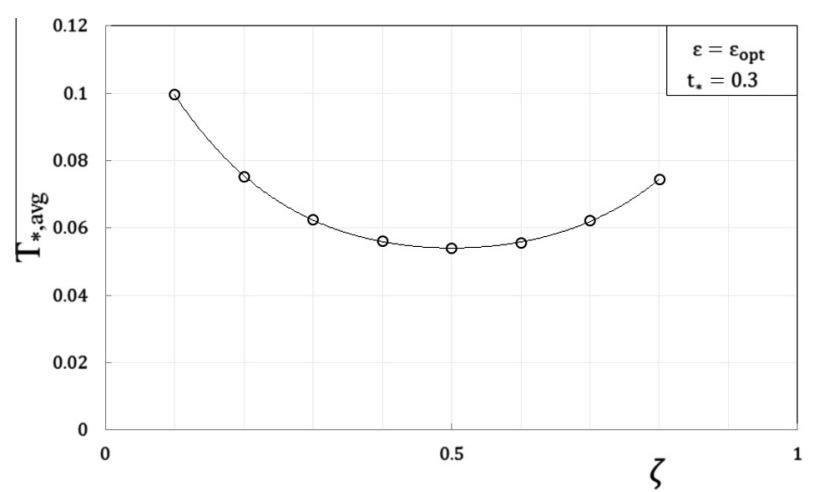

Fig. 5. The effect of the spacing between the spirals $(\zeta)$ on the average temperature of the conducting surroundings.
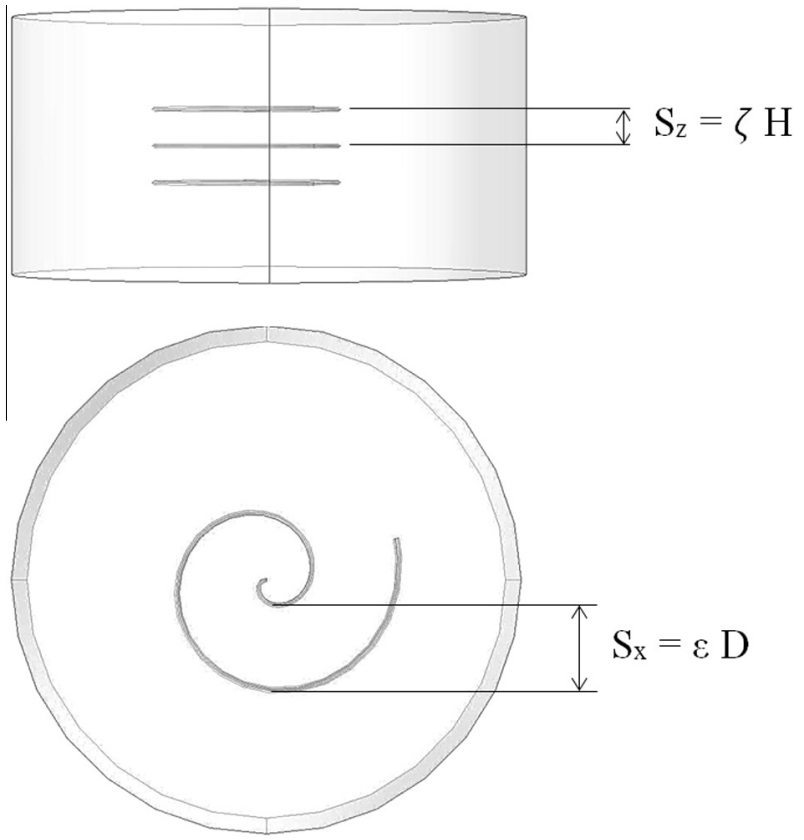

Fig. 6. The geometry of three spiral pipes embedded in a cylindrical conducting volume.

other $t_{x}$ values we found that $\zeta_{\text {opt }}$ does not vary with $t_{*}$ in the range $t_{*}=0.1-1$

Next, we optimized the thermal coupling between the cylindrical volume and three spiral pipes embedded in the conducting domain, as shown in Fig. 6. The total volume of the three spiral pipes is the same as the total volume of the two spiral pipes studied earlier. In the case of Fig. 6, we are interested in finding the optimum spacings ( $\varepsilon$ and $\zeta$ ) that correspond to minimum average temperature of the cylindrical volume $V_{c}$. Fig. 7 shows the effect of varying the spacing between the spiral turns $\varepsilon$ on the average temperature of the cylindrical volume for three different values of $\zeta$. There is an optimum spacing between the spiral turns $\left(\varepsilon_{o p t}=0.3\right.$, in this case) that corresponds to the minimum average temperature of the cylindrical volume. This optimum spacing $\varepsilon_{o p t}$ does not depend on $\zeta$. By repeating this work for other $t_{*}$ values, we found that $\varepsilon_{o p t}$ does not depend on time $t_{*}$.

The effect of the spacing between the three spirals on the dimensionless temperature $T_{*}$ can be determined by varying the value of $\zeta$ after fixing the value of $\varepsilon$ to $\varepsilon_{o p t}$, as shown in Fig. 8 . The optimum value of $\zeta$ that corresponds to the minimum average

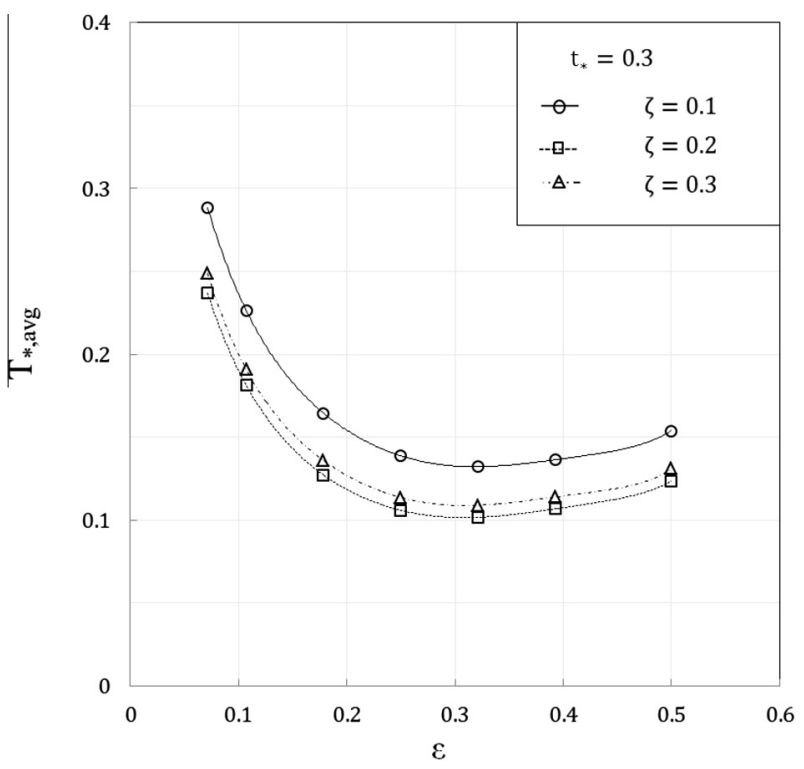

Fig. 7. The effect of the spacing between the spiral turns $(\varepsilon)$ on the average temperature of the cylindrical domain.

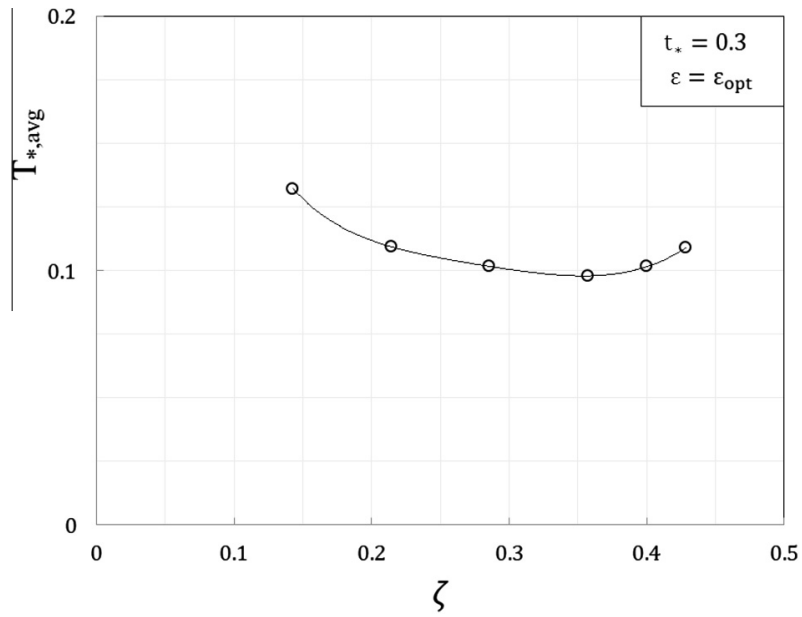

Fig. 8. The effect of the spacing between the spirals $(\zeta)$ on the average temperature of the cylindrical domain.

temperature of the cylindrical volume is 0.35 . Furthermore, we found that $\zeta_{\text {opt }}$ does not vary with $t_{*}$ in the range $t_{*}=0.1-1$.

We extended this study by relaxing the assumption that $H=D / 2$ in order to determine the effect of varying the dimensions of the conducting cylindrical volume on the performance of the thermal coupling. We started with two spiral pipes embedded in the conducting volume of size $H=D$. Fig. 9 shows the effect of varying the spacing between the spiral turns $\varepsilon$ on the average temperature for three different values of $\zeta$. We further found that the optimum spacing between the spiral turns is $\varepsilon_{o p t}=0.3$ and that this value does not vary with $t_{*}$ in the range $t_{*}=0.1-1$. Fig. 10 shows the effect of varying the spacing between the two spirals $\zeta$. The optimum spacing between the two spirals is $\zeta=0.5$.

For three spirals, Fig. 11 shows that the optimum spacing between the spiral turns is $\varepsilon_{\text {opt }} \cong 0.3$. By repeating this work for other $t_{*}$ values, we also found that $\varepsilon_{o p t}$ does not vary with $t_{*}$. The effect of the spacing between the spirals is shown in Fig. 12. The figure shows that the optimum spacing between the three spirals is $\zeta_{\text {opt }}=0.34$ when $t_{*}=0.3$. Furthermore, we found that $\zeta_{\text {opt }}$ does 


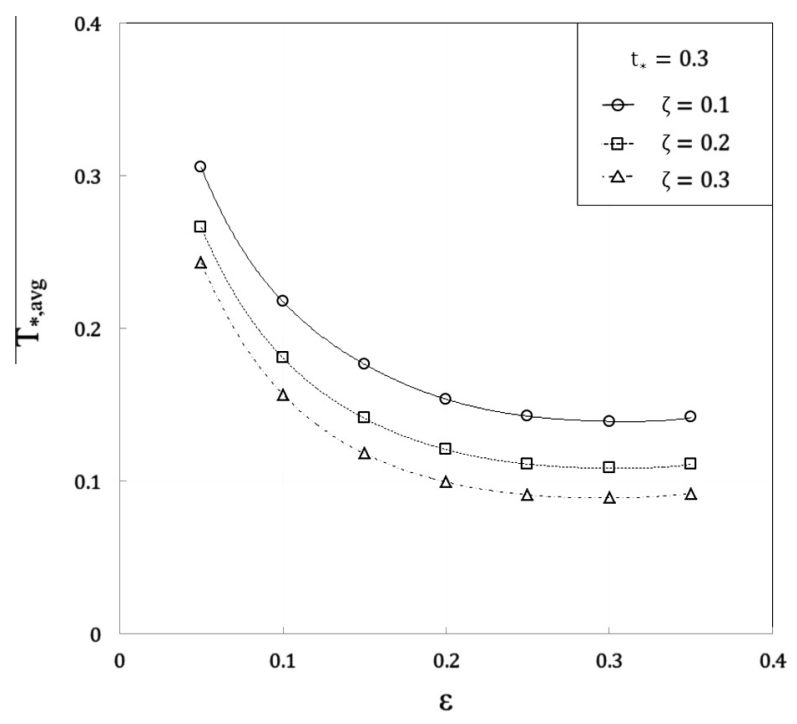

Fig. 9. The effect of the spacing between the spiral turns $(\varepsilon)$ on the average temperature of the cylindrical domain when $H=D$.

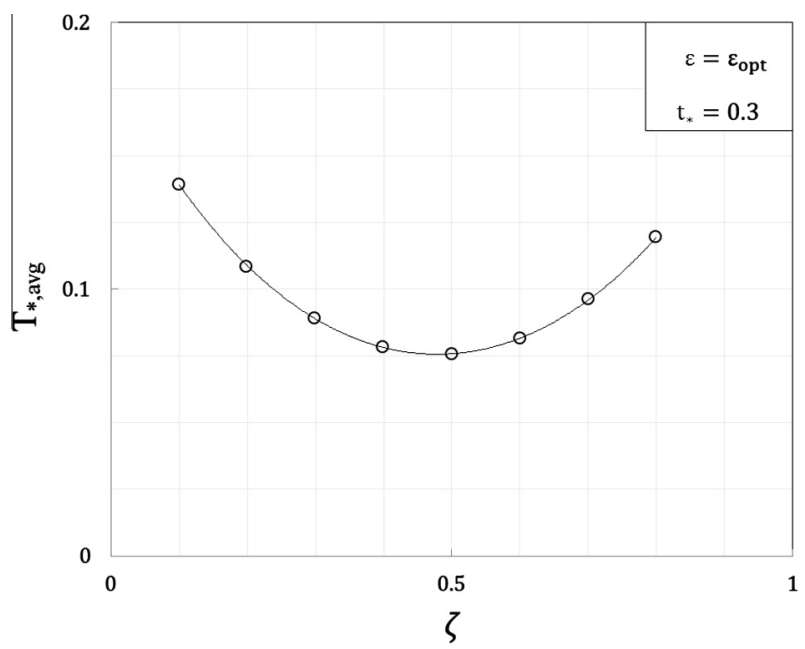

Fig. 10. The effect of the spacing between two spirals $(\zeta)$ on the average temperature of the cylindrical domain when $H=D$.

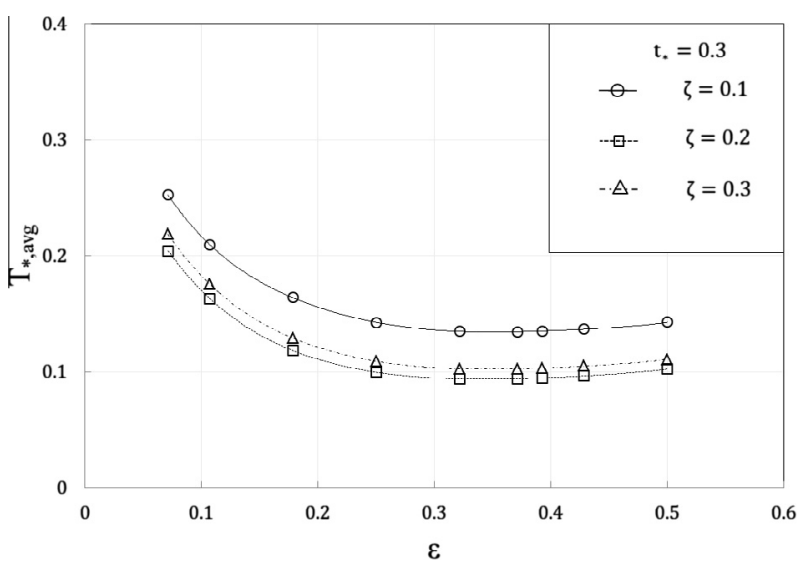

Fig. 11. The effect of the spacing between the spiral turns $(\varepsilon)$ on the average temperature of the cylindrical domain when $H=D$.

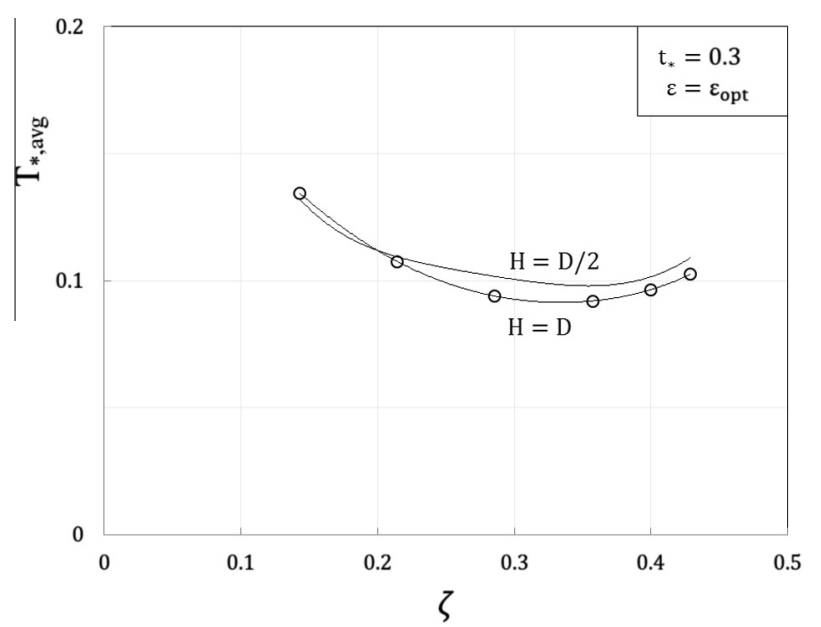

Fig. 12. The effect of the spacing between the spirals $(\zeta)$ on the average temperature of the cylindrical domain when $H=D$.

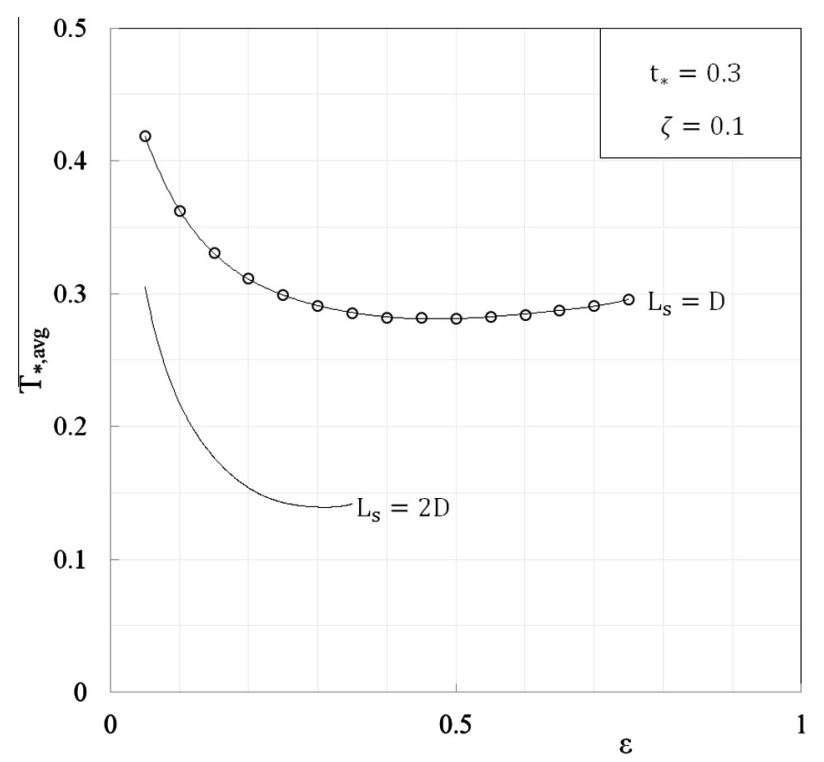

Fig. 13. The effect of changing the length of the heat exchanger with two spirals when $H=D$.

not vary with $t_{x}$ in the range $t_{x}=0.1-1$. The optimal design and performance for $H=D / 2$ is essentially the same for $H=D$, cf. Fig. 8 .

So far, the length of the heat exchanger was held fixed at $L_{s}=2 D$. We relaxed this constraint, and repeated the numerical simulations for $L_{s}=D$ when $H=D$. The comparison shown in Fig. 13 shows that when the heat exchanger is shorter the optimal horizontal spacing $(\varepsilon)$ is larger, and the performance is poorer ( $T_{*}$ avg is larger).

\section{Concluding remarks}

By comparing Figs. 4 and 5 with Figs. 9 and 10, we conclude that both the optimum spacing between the spiral turns and the optimum spacing between the spirals are independent of the size of the conducting volume. This observation is valid for configurations with two and three spiral pipes. Worth noting is that the optimum spacing between the spirals $\zeta_{\text {opt }}$ is almost equal to inverse of the number of spirals. For example, where for two spirals $\zeta_{\text {opt }} \cong 1 / 2$, and for three spirals $\zeta_{\text {opt }} \cong 1 / 3$.

The chief conclusion is that the spiral geometry optimized for maximum volumetric heat transfer is robust with respect to changes 
in several physical parameters. Moreover, the optimum spiral geometry that corresponds to minimum average temperature $T_{*, \text { avg }}$ does not vary with time $t_{*}$ in the range $t_{*}=0.1-1$. The optimum spacing between the spiral turns $\varepsilon$ is larger when the heat exchanger is shorter, and the performance is poorer.

The method developed in this paper can be extended to the optimization of geometry in more complicated configurations of ground coupled heat exchangers. For example, considering a helical spiral pipe embedded in a cylindrical conducting volume and study the effect of varying the radial and axial pitch on the heat transfer performance or conducting similar study for staggered arrangement of spiral pipes.

\section{Conflict of interest}

None declared.

\section{Acknowledgment}

Mr. Alalaimi's work was supported by Kuwait University. Profs. Lorente and Bejan's work was sponsored by the National Science Foundation and the National Renewable Energy Laboratory.

\section{References}

[1] A. Bejan, Advanced Engineering Thermodynamics, second ed., Wiley, New York, 1997.

[2] A. Bejan, S. Lorente, The constructal law of design and evolution in nature, Philos. Trans. R. Soc. B 365 (2010) 1335-1347.

[3] A. Bejan, S. Lorente, The constructal law and the evolution of design in nature, Phys. Life Rev. 8 (2011) 209-240.

[4] B. Sanner, C. Karytsas, D. Mendrinos, L. Rybach, Current status of ground source heat pumps and underground thermal energy storage in Europe, Geothermics 32 (2003) 579.

[5] I. Dincer, M.A. Rosen, Thermal Energy Storage: Systems and Applications, Wiley, Chichester, 2002.

[6] F.H. Cocks, Energy Demand and Climate Change, Wiley, Weinhem, 2009.
[7] M.H. Abbaspour-Fard, A. Gholami, M. Khojastehpour, Evaluation of an Earthto-air heat exchanger for the North-East of Iran with semi-arid climate, Int. J. Green Energy 8 (2011) 499.

[8] K.S. Lee, Modeling on the performance of standing column wells during continuous operation under regional groundwater flow, Int. J. Green Energy 8 (2011) 474

[9] G. Florides, S. Kalogirou, Ground heat exchangers - a review of systems, models and applications, Renewable Energy 32 (2007) 2461.

[10] See http://cipco.apogee.net/ for Geothermal Heat Pump Systems 2005, CIPCO Energy Library.

[11] T. Katsura, K. Nagano, S. Takeda, Method of calculation of the ground temperature for multiple ground heat exchangers, Appl. Therm. Eng. 28 (1415) (2008) 1995.

[12] H. Demir, A. Koyun, G. Temir, Heat transfer of horizontal parallel pipe ground heat exchanger and experimental verification, Appl. Therm. Eng. 29 (2009) 224.

[13] Y. Nam, R. Ooka, Numerical simulation of ground heat and water transfer for groundwater heat pump system based on real-scale experiment, Energy Build. 42 (2010) 69.

[14] H. Esen, M. Inalli, M. Esen, Numerical and experimental analysis of a horizontal ground-coupled heat pump system, Build. Environ. 42 (2007) 1126.

[15] Y. Shang, S. Li, H. Li, Analysis of geo-temperature recovery under intermittent operation of ground-source heat pump, Energy Build. 43 (2011) 935.

[16] D. Bozis, K. Papakostas, N. Kyriakis, On the evaluation of design parameters effects on the heat transfer efficiency of energy piles, Energy Build. 43 (2011) 1020.

[17] V. Khalajzadeh, G. Heidarinejad, J. Srebric, Parameters optimization of a vertical ground heat exchanger based on response surface methodology, Energy Build. 43 (2011) 1288.

[18] P. Cui, H. Yang, Z. Fang, Numerical analysis and experimental validation of heat transfer in ground heat exchangers in alternative operation modes, Energy Build. 40 (2008) 1060.

[19] H. Esen, M. Inalli, M. Esen, K. Pihtili, Energy and exergy analysis of a groundcoupled heat pump system with two horizontal ground heat exchangers, Build. Environ. 42 (2007) 3606.

[20] J. Darkwa, G. Kokogiannakis, C.L. Magadzire, K. Yuan, Theoretical and practical evaluation of an earth-tube (E-tube) ventilation system, Energy Build. 43 (2011) 728.

[21] E.K. Akpinar, A. Hepbasli, A comparative study on exergetic assessment of two ground-source (geothermal) heat pump systems for residential applications, Build. Environ. 42 (2007) 2004.

[22] K. Woods, A. Ortega, The thermal response of an infinite line of open loop wells for ground coupled heat pump systems, Int. J. Heat Mass Transfer 54 (2011) 5574.

[23] http://www.comsol.com. 